\title{
PLANTAS INVASORAS DE VÁRZEA NO ESTADO DE SÃO PAULO
}

\section{RESUMO}

As plantas invasoras que ocorrem em uma determinada cultura, constituem sério problema, tendo em vista a concorrência em água, luz e nutrientes que promove com a planta cultivada, determinando uma sensivel queda da produtividade da lavoura. O levantamento e reconhecimento dessas plantas, além do estudo biológico, permitem um controle mais eficiente e económico.

A coleta do material botânico foi realizada através de visitas em áreas de várzeas cultivadas e em três épocas distintas do ano, com a finalidade de se levantar o maior número possível de espécies invasoras. A fenologia, o modo ou modos de reprodução, frequência e ciclo são elementos indispensáveis para um controle racional e efetivo.

Apesar do maior número de espécies pertencerem as famílias Compositae e Gramineae, as espécies de maior importância econômica estão abrigadas também nas famílias Cyperaceae, Lythraceae, Onagraceae, Polygonaceae, Pontederiaceae e Portulacaceae.

PALAVRAS CHAVES: Vá rzea, Planta Invasora, Levantamento, Biologia.

\author{
C. ARANHA*, H.F. LEITÃO F".** \& \\ R.M.PIO*** \\ * Pesquisador Científico. Seção de Botânica \\ Econômica do IAC. C.P.28 13.100 Campinas. \\ Bolsista do CNPq. \\ ** Professor Departamento de Botânica - UNI- \\ CAMP. \\ *** Biologista Seção de Botânica Econômica do \\ IAC - Contratada pelo Convênio IAC-EMBRAPA- \\ BAGs.
}

\section{SUMMARY}

Due to competition for water, light and nutrients the weeds are serious problems for certain kind of crops. They are responsible for the reduction in production. The survey and the recognition of different weeds and the their biological study permit a more efficient and economical way of control.

The botanical material was collected in lowland crops during three different periods of the year. The purpose was to collect as many different species of weeds as possible. The phenology, the mode of reproduction, the frequency and cycle of weed species are important factors for the rational and effective control.

Although the greatest amount of weed species collected belong to the Compositae and Gramineae, the most economically important species belong to the following families: Cyperaceae, Lythraceae, Onagraceae, Polygonaceae, Pontederiaceae and Portulacaceae.

KEY WORDS: Lowland, weed. survey, biolology

\section{INTRODUÇÃO}

As plantas invasoras representam 
sério inconveniente para as culturas pelos múltiplos prejuízos que ocasionam, quer dificultando e onerando os tratos culturais ou determinando perdas nas colheitas pela concorrência por água, nutrientes e luz que exercem às plantas cultivadas $(1,9,10,11,12,17,20,21$, $24,25)$. Desta forma, todos os esforços realizados no sentido de minorar a incidência de plantas invasoras são válidas e representam valiosa colaboração para - aumento da produtividade das lavouras, objetivo primordial do empreendimento agrícola.

A flora das várzeas é bastante típica e obedece uma uniformidade acentuada. Afora este fato, a amplitude geográfica das plantas de várzea é muito grande, obviamente pela presença, em seu habitat, de um fator comum que é a elevada umidade do solo. Este fato implica que a grande maioria das plantas que ocorrem no Estado de São Paulo também o fazem nos Estados limítrofes, e de uma forma geral a sua variação qualitativa e quantitativa obedece a mesma sequência para toda região centro-sul do Brasil, permitindo extrapolações válidas para extensas áreas do território brasileiro $(1,24,25)$.

Neste trabalho são apresentadas algumas características das principais plantas invasoras de várzeas do Estado de São Paulo que ocorrem não apenas em áreas alagadas como também ao longo de canais de irrigação e regiões fronteiriças.

\section{MATERIAIS E MÉTODOS}

Os materiais botânicos estudados foram co- lhidos através de viagens realizadas pelas culturas de várzeas do Estado de São Paulo em três épocas distintas do ano. Para racionalizar essas viagens, o Estado foi convencionalmente dividido em seis regiôes a saber: Litoral, Vale do Paraíba, Planalto, Mojiana, Noroeste e Araraquarense e cada uma dessas regiôes foi subdividida em função de sua extensão facilitando, dessa forma, a localização das culturas.

Para estudo do ciclo vegetativo cada espécie foi semeada em casa de vegetação e acompanhado todo o seu desenvolvimento até a frutificação, exceção feita às espécies cujos ciclos já tinham sido estudados quando da realização do Projeto "Plantas Invasoras de Culturas no Estado de São Paulo".

No sentido de se estabelecer parâmetros capazes de definirem freqüência baix a, regular e alta, utilizou-se o critério de observação em toda área cultivada, sendo que, essa observação foi efetuada por dois botânicos que estabeleceram independentemente os diferentes graus de ocorrência obedecendo aos percentuais a seguir expostos.

Percentual da freqüência da espécie invasora com relação a área total da cultura.

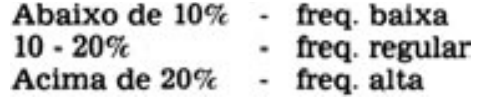

A terminologia botânica utilizada neste trabalho consta integralmente do Dicionário de Botânica - Font Quer (15).

\section{RESULTADOS E DISCUSSÃO}

As plantas constantes deste levantamento acham-se distribuídas em ordem alfabética de famílias, gêneros e espécies. De cada planta são apresentados o nome científico, os nomes vulgares, freqüência, ciclo, fenologia e modo ou modos de reprodução. 
RESULTADOS DO LEVANTAMENTO

\begin{tabular}{|c|c|c|c|c|c|c|}
\hline NOME CIENTIFICO & NOME VULGAR & FREQUÊNCIA & ciclo & FLORESC. & FRUTIF. & REPRODUÇĀO \\
\hline $\begin{array}{l}\text { ALISMATACEAE } \\
\text { Echinodorus ellipticus (Mart.) Mich } \\
\text { Echinodorus grandiflorus Mich } \\
\text { Sagittaria montevidensis Cham. et Schlecht } \\
\text { Sagittaria sellowiana Kunt }\end{array}$ & $\begin{array}{l}\text { Chapéu-de-couro } \\
\text { Chapéu-de-couro } \\
\text { Flecha } \\
\text { Flecha }\end{array}$ & $\begin{array}{l}\text { Baixa } \\
\text { Baixa } \\
\text { Baixa } \\
\text { Baixa }\end{array}$ & $\begin{array}{l}\text { Anual } \\
\text { Anual } \\
\text { Anual } \\
\text { Anual }\end{array}$ & $\begin{array}{l}\text { Nov-Jan } \\
\text { Nov-Jan } \\
\text { Nov-Jan } \\
\text { Nov-Fev }\end{array}$ & $\begin{array}{l}\text { Jan-Mar } \\
\text { Jan-Mar } \\
\text { Jan-Abr } \\
\text { Jan-Abr }\end{array}$ & $\begin{array}{l}\text { Sementes } \\
\text { Sementes } \\
\text { Sementes } \\
\text { Sementes }\end{array}$ \\
\hline $\begin{array}{l}\text { AMARANTHACEAE } \\
\text { Alternanthera philoxeroides (Mart.) Griseb } \\
\text { A maranthus deflexus } \mathrm{L} \text {. } \\
\text { A maranthus hybridus } \mathrm{L} . \\
\text { A maranthus viridis } \mathrm{L} . \\
\text { Asclepias curassavica } \mathrm{L} \text {. }\end{array}$ & $\begin{array}{l}\text { Carrapicho-de-brejo } \\
\text { Carurú } \\
\text { Carurú } \\
\text { Carurú } \\
\text { Oficial-de-sala, paina-de- } \\
\text { sapo }\end{array}$ & $\begin{array}{l}\text { Regular } \\
\text { Regular } \\
\text { Regular } \\
\text { Regular } \\
\text { Regular }\end{array}$ & $\begin{array}{l}\text { Perene } \\
\text { Anual } \\
\text { Anual } \\
\text { Anual } \\
\text { Anual }\end{array}$ & $\begin{array}{l}\text { Jan-Fev } \\
\text { Set-Dez } \\
\text { Set-Dez } \\
\text { Set-Dez } \\
\text { Abr-Mai }\end{array}$ & $\begin{array}{l}\text { Fev-Abr } \\
\text { Dez-Mar } \\
\text { Nov-Mar } \\
\text { Nov-Mar } \\
\text { Jun-Ago }\end{array}$ & $\begin{array}{l}\text { Semen. e estolōes } \\
\text { Sementes } \\
\text { Sementes } \\
\text { Sementes } \\
\text { Sementes }\end{array}$ \\
\hline $\begin{array}{l}\text { BORRAGINACEAE } \\
\text { Heliotropium indicum } \mathrm{L} .\end{array}$ & Borragem & Regular & Anual & Jan-Mar & Mar-Mai & Sementes \\
\hline $\begin{array}{l}\text { BUTOMACEAE } \\
\text { Hydrocleis humboldtii Endl. } \\
\text { Limnocharis flava Rich. }\end{array}$ & $\begin{array}{l}\text { Gôlfo } \\
\text { Gôlfo }\end{array}$ & $\begin{array}{l}\text { Baixa } \\
\text { Baixa }\end{array}$ & $\begin{array}{l}\text { Perene } \\
\text { Perene }\end{array}$ & $\begin{array}{l}\text { Out-Dez } \\
\text { Out-Dez }\end{array}$ & $\begin{array}{l}\text { Dez-Fev } \\
\text { Dez-Fev }\end{array}$ & $\begin{array}{l}\text { Semen. e rizomas } \\
\text { Semen. e rizomas }\end{array}$ \\
\hline $\begin{array}{l}\text { CAMPANULACEAE } \\
\text { Isotoma longiflora Presl. }\end{array}$ & $\begin{array}{l}\text { Arrebenta-boi, arrebenta- } \\
\text { cavalo, cega-ôlho }\end{array}$ & Baixa & Perene & Set-Nov & Nov-Jan & Sementes \\
\hline $\begin{array}{l}\text { CAPPARIDACEAE } \\
\text { Cleome spinosa } \mathrm{L} .\end{array}$ & Mussambé-de-espinho & Regular & Anual & Out $-\mathrm{Fev}$ & Dez-Mar & Sementes \\
\hline $\begin{array}{l}\text { CAR YOPHYLLACEAE } \\
\text { Drymaria condata (L) Willd. } \\
\text { Stellaria media }(\mathrm{L}) \text { Cyrill }\end{array}$ & $\begin{array}{l}\text { Mastruço-do-brejo } \\
\text { Esparguta }\end{array}$ & $\begin{array}{l}\text { Regular } \\
\text { Baixa }\end{array}$ & $\begin{array}{l}\text { Anual } \\
\text { Anual }\end{array}$ & $\begin{array}{l}\text { Out-Dez } \\
\text { Out-Dez }\end{array}$ & $\begin{array}{l}\text { Jan-Fev } \\
\text { Jan-Fev }\end{array}$ & $\begin{array}{l}\text { Sementes } \\
\text { Sementes }\end{array}$ \\
\hline $\begin{array}{l}\text { COMMELINACEAE } \\
\text { Commelina agraria Kunth. } \\
\text { Commelina elegans H.B.K. } \\
\text { Commelina nudiflora L. } \\
\text { Tradescantia elongata G.F.W. Mey }\end{array}$ & $\begin{array}{l}\text { Trapoeraba } \\
\text { Trapocraba } \\
\text { Trapocraba } \\
\text { Trapocraba }\end{array}$ & $\begin{array}{l}\text { Baixa } \\
\text { Regular } \\
\text { Regular } \\
\text { Baixal }\end{array}$ & $\begin{array}{l}\text { Perene } \\
\text { Perene } \\
\text { Perene } \\
\text { Perene }\end{array}$ & $\begin{array}{l}\text { Nov-Jan } \\
\text { Out-Dez } \\
\text { Out-Dez } \\
\text { Out-Dez }\end{array}$ & $\begin{array}{l}\text { Dez-Mar } \\
\text { Dez-Fev } \\
\text { Dez-Fev } \\
\text { Dez-Fev }\end{array}$ & $\begin{array}{l}\text { Semen. e estolōes } \\
\text { Semen. e estolōes } \\
\text { Semen. c estolōes } \\
\text { Semen. e estolōes }\end{array}$ \\
\hline
\end{tabular}




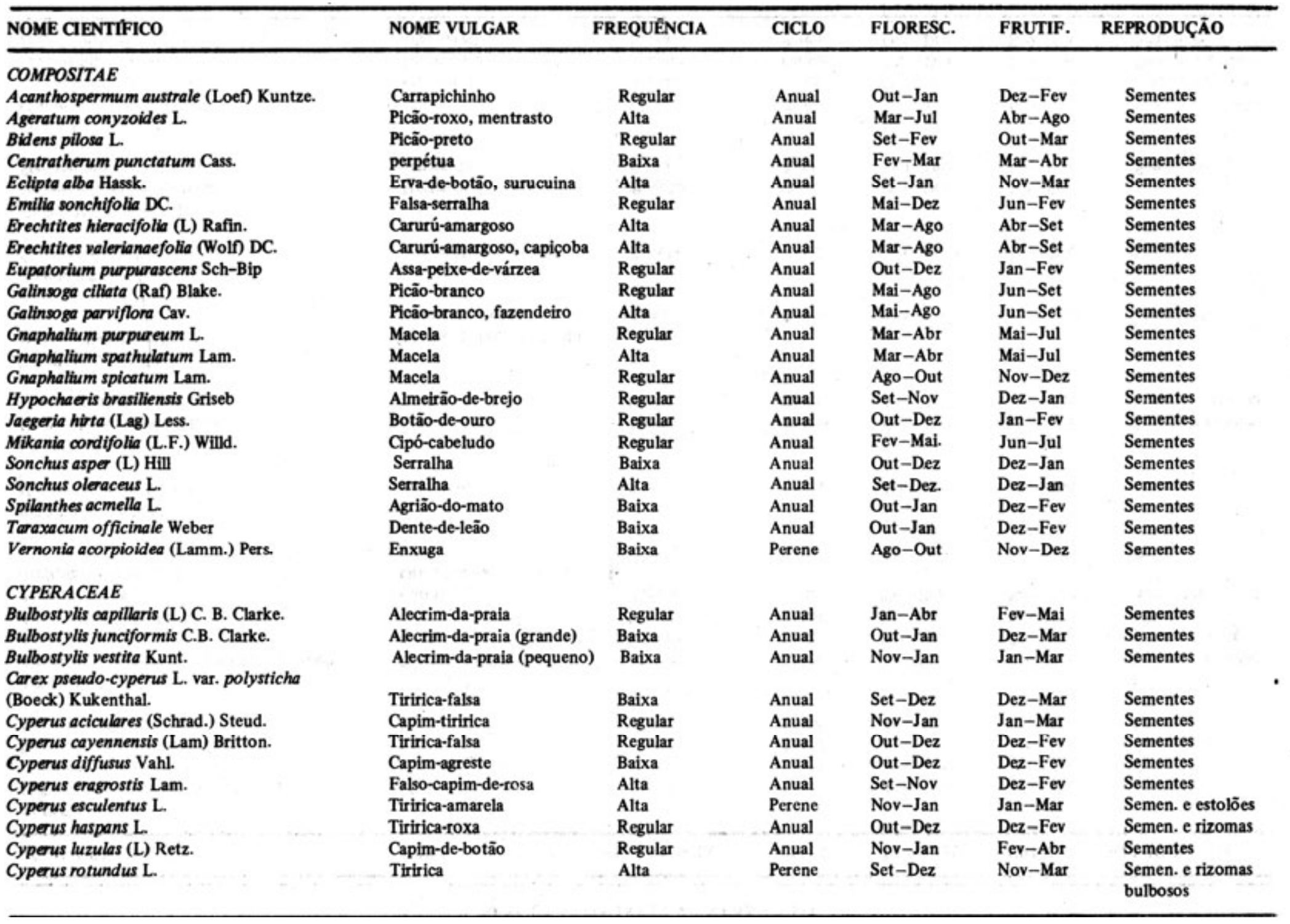


Cyperus sesquiflorus (Torrey) Mattf. et

Kukenthal

Cyperus virens Michx.

Fimbristylis autumnalis (L) Roem. et Schult. Fuirena robusta Kunth.

Eleocharis elegans (H.B.K.) R. et S.

Eleocharis filiculmis Kunth.

Eleocharis fistulosa (Poir) Link

Eleocharis nodulosa (Roth.) Roem. et Schult.

Rhynchospora corymbosa (L) Britton.

Rhynchospora exaltata Kunth.

Rhynchospora marisculus Lindl. et Nees.

Scleria bracteata Cav.

Scleria latifolia Swartz.

\section{ERIOCAULACEAE}

Eriocaulon helichrysoides Steud.

Eriocaulon sellowianum Kunth.

Saepalanthus xeranthemoides Mart.

\section{EUPHORBIACEAE}

Euphorbia pilulifera L.

Euphorbia prostrata Ait.

Phyllanthus niruri $\mathrm{L}$.

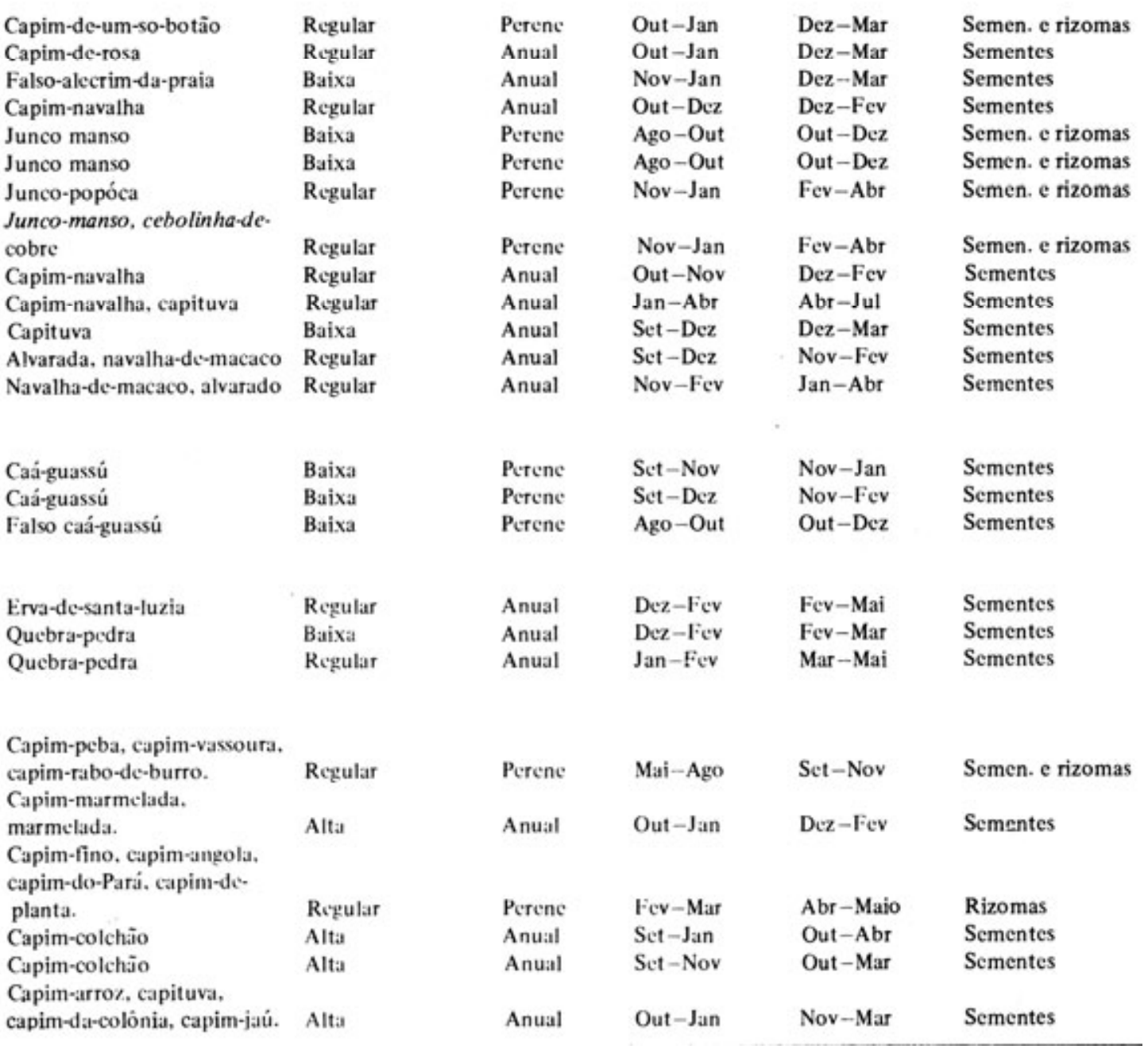

\section{GRAMINEAE}

Andropogon bicornis L.

Brachiaria plantaginea (Link) Hitch.

Brachiaria purpurascens Henr.

Digitaria horizontalis Wi!11.

Digitaria sanguinalis (L) Scop.

Echinochloa colonum (L) LInk. 


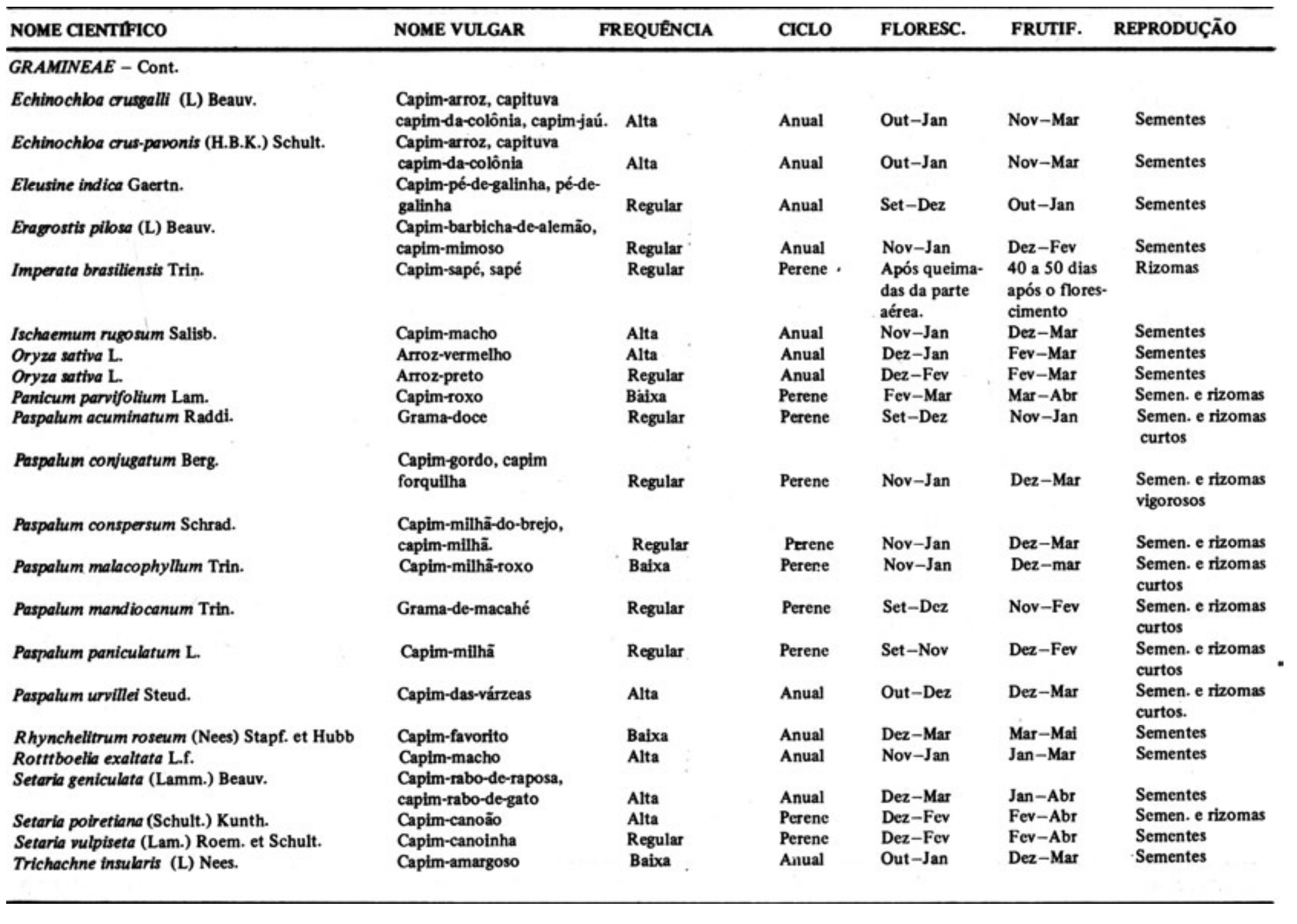

Capim-arroz, capituva

Alta

Capim-pé-de-galinha, pé-de-

Capim-barbicha-de-alemão,

capim-mimoso 


\begin{tabular}{|c|c|c|c|c|c|c|}
\hline NOME CIENTIFICO & NOME VULGAR & FREQUÊNCIA & CicLo & FLORESC. & FRUTIF. & REPRODUÇĀo \\
\hline \multicolumn{7}{|l|}{ HYDROPHYLLACEAE } \\
\hline Hydrolea spinosa $\mathrm{L}$ & Carqueja-do-brejo & Regular & Perene & Mai-Jul & Ago-Set & Sementes \\
\hline \multicolumn{7}{|l|}{ HYPOXIDACEAE } \\
\hline Hypoxis decumbens $\mathrm{L}$. & Falsa-tiririca & Baixa & Anual & Set-Dez & Nov-Fev & Sementes \\
\hline \multicolumn{7}{|l|}{ JUNCACEAE } \\
\hline Juncus communis Meyer & Junco & Regular & Anual & Abr-Jun & Mai-Ago & Sementes \\
\hline Juncus densiflorus Humb. et Kunth & Junco & Baixa & Anual & Abr-Jun & Jun-Set. & Sementes \\
\hline Juncus sellowiana Kunth. & Junco & Regular & Anual & Abr-Ago & Jun-Out & Sementes \\
\hline \multicolumn{7}{|l|}{ LABIATAE } \\
\hline Hyptis brevipes Poit. & hiptis & Regular & Anual & Fev-Mar & Abr-Mai & Sementes \\
\hline Leonotis nepetaefolia $\mathrm{R}$. Br. & Cordão-de-frade & Regular & Anual & Out-Dez & Jan-Fev & Sementes \\
\hline Leonurus sibiricus L. & Rubim & Regular & Anual & Out-Dez & Jan-Fev & Sementes \\
\hline \multicolumn{7}{|l|}{ LEGUMINOSAE } \\
\hline Mimosa invisa Mart. & Dormideira & Regular & Perene & Fev-Mar & Abr-Jun & Sementes \\
\hline Mimosa pudica $\mathrm{L}$. & Sensitiva & Regular & Perene & Sct-Dez & Mar-Abr & Sementes \\
\hline Aeschynomene selloi Vog. & Paricá & Regular & Perene- & Dez-Fev & Fcv-Mar & Sementes \\
\hline Desmodium barbatum (L) Benth. & Carrapicho-beiço-dc-boi & Baixa & Anual & Abr-Jun & Jun-Set & Sementes \\
\hline Sesbania exasperata H.B.K. & Cássia-do-brejo & Regular & Perene & Jan-Mar & $\mathrm{Abr}-\mathrm{Mai}$ & Sementes \\
\hline \multicolumn{7}{|l|}{ LYTHRACEAE } \\
\hline Cuphea carthagenensis (Jacq.) Macbr, & Sete-sangrias & Alta & Anual & Dez-Mar & Jan-Abr & Sementes \\
\hline Cuphea mesostemon Koehne & Sete-sangrias & Baixa & Anual & Dez-Mar & Jan-Abr & Sementes \\
\hline \multicolumn{7}{|l|}{$M A L V A C E A E$} \\
\hline Malvastrum coromandelianum (L) Gurcke & Falsa-guanxuma & Baixa & Anúal & Dez-Mar & Fev-Mai & Sementes \\
\hline Sida rhombifolia L. & Guanxuma, guaxima & Baixa & Anual & Dez-Abr & Jan-Mai & Sementes \\
\hline \multicolumn{7}{|l|}{ MELASTOMATACEAE } \\
\hline Aciotis paludosa Triana & Falsa-quaresmeira-do-brejo & Baixa & Perene & Nov-Fev & Jan-Mar & Sementes \\
\hline Tibouchina gracilis (Borg.) Cogn. & Quaresmeira-do-brejo & Regular & Perene & Set-Dez & Dez-Fev & Sementes \\
\hline \multicolumn{7}{|l|}{ MOLLUGINACEAE } \\
\hline Mollugo verticillata $\mathrm{L}$. & - & Regular & Anual & Out-Fev & Dez-Mar & Sementes \\
\hline
\end{tabular}




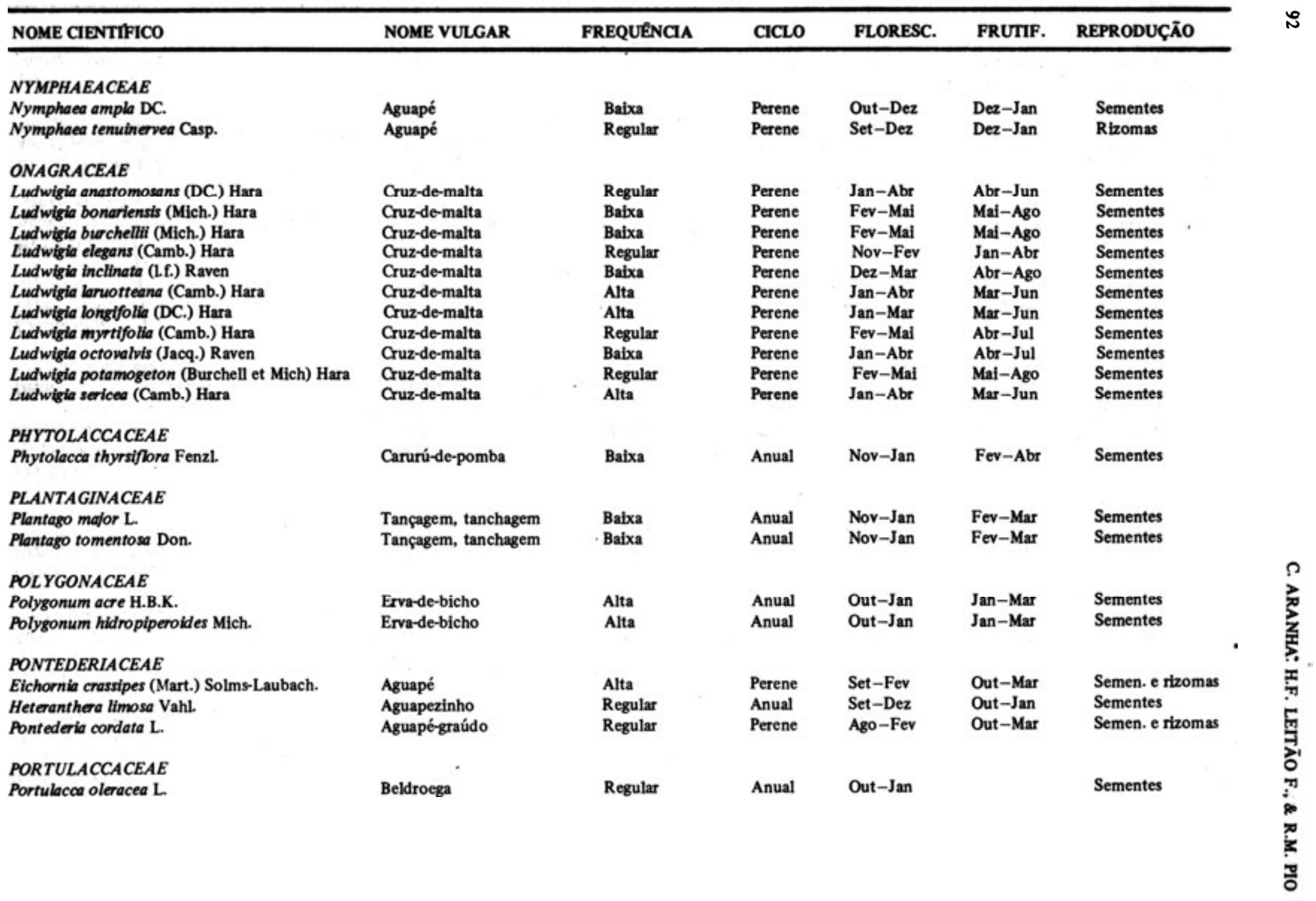




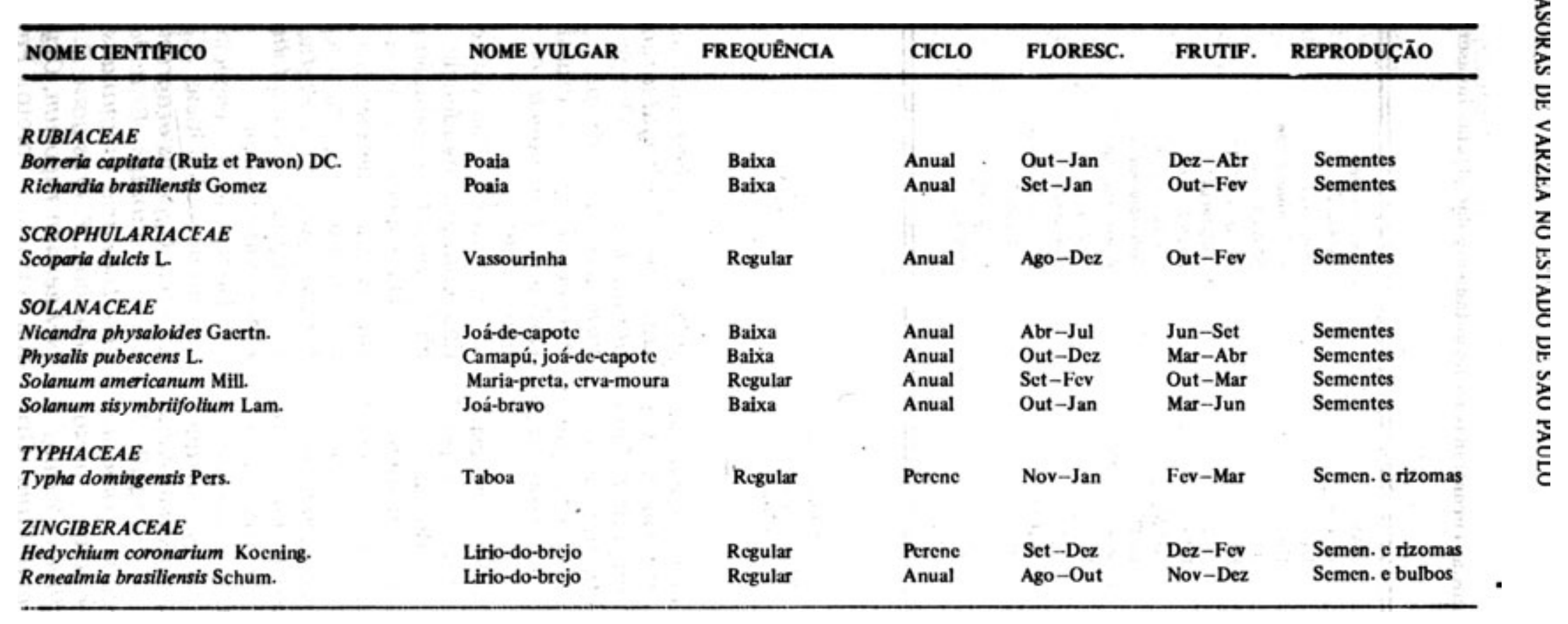


QUADRO 1. Relação das famí lias encontradas no levantamento das plantas invasoras de culturas de várzea.

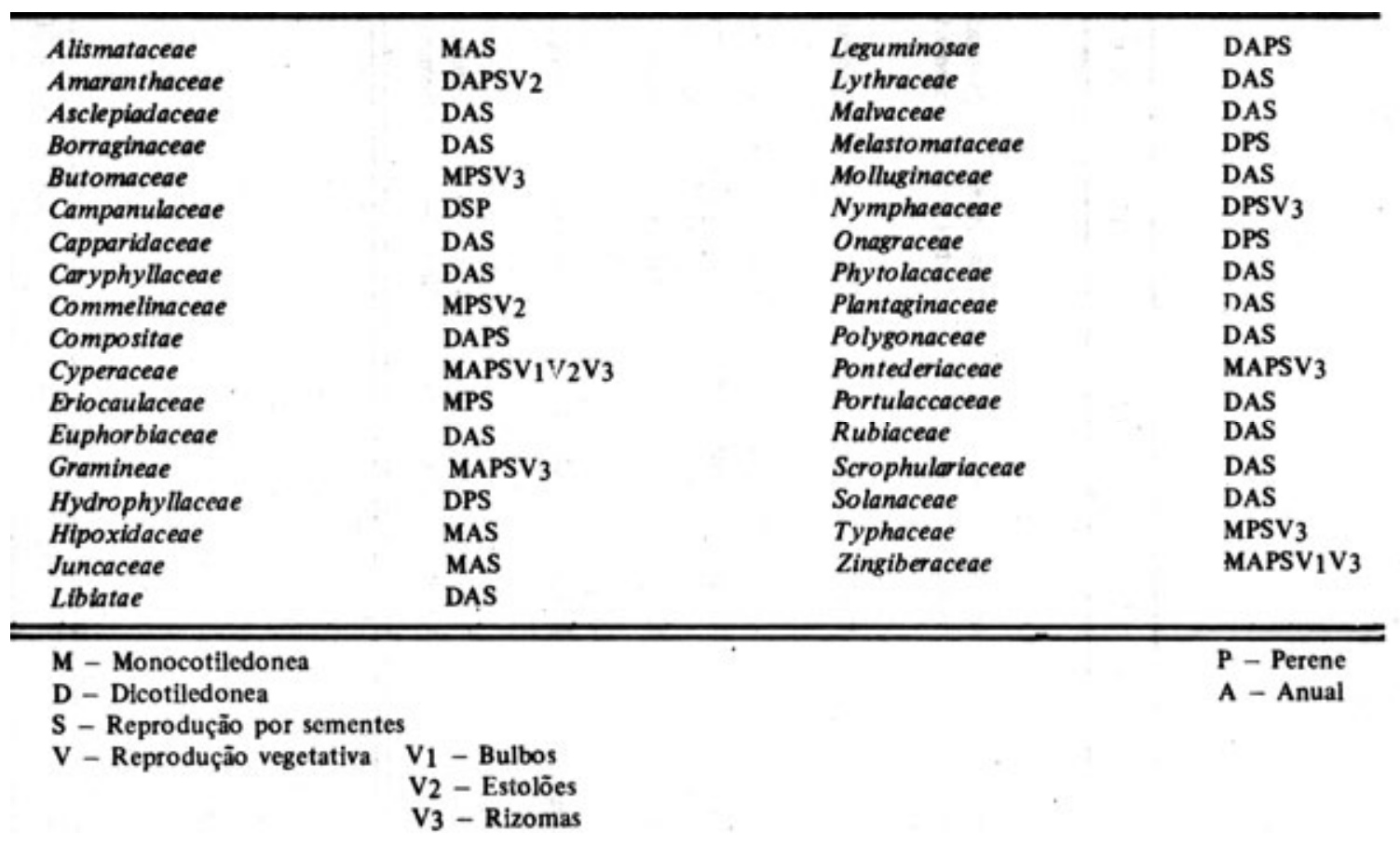

O levantamento e identificação das plantas invasoras de culturas de várzeas mostrou-nos a presença de 35 famílias, entre mono e dicotiledóneas, conforme exposto no quadro 1, onde também são fornecidos os ciclos e modos de reprodução.

Apesar do maior número de es pé cies pertencerem às famílias Compositae e Gramineae $(3,8,18,19)$, as mais importantes por sua alta incidência, competição e danos causados a estas culturas são: Ageratum conyzoides, Eclipta alba, Erechtites hieracifolia, E. valerianaefolia, Galinsoga parviflora, Gnaphalium spathulatum, Sonchus oleraceus(Compositae), Cyperuseragrostis, C. esculentus (Cyperaceae); Brachiariaplantaginea, Digitaria sanguinalis, Echinochloa colonum, E. crus-galli, E. crus-pavonis, Ischaemum rugosum, Oryza sativa (arroz vermelho e preto), Paspalum urvillei, Rottboelia exaltata, Setaria geniculata, S. poiretiana (Gra- mineae), Cuphea carthagenensis (Lythraceae); Ludwigia laruotteana, L. iongifolia, L. sericea (Onagraceae); Polygonum acre, $P$. hidropiperoides (Polygonaceae); Eichornia crassipes (Pontederiaceae); Portulacca oleracea (Portulaccaceae).

A maioria das es pécies en contradas, com alta incidência, apresentaram ciclo anual e reprodução por sementes.

Das espécies de ciclo perene, mostraram-se mais agresivas e competitivas as seguintes: Cyperus esculentus, C. rotundus, Setaria poiretiana, Ludwigia laruotteana, L. longifolia, L. sericea, Eichornia crassipes.

Dentre estas espécies agressivas, são monocotiledoneas $(4,5,6,7,16)$ as seguintes: Cyperus eragrostis, C. esculentus, C. rotundus, Brachiaria plantaginea,Digitaria sanguinalis, Echinochloa colonum, E. crus-galli, E. crus-pavonis, Ischaemum rugosum, Oryza sativa (arroz vermelho e preto), Paspalum urvil- 
lei, Rottboelia exaltata, Setaria geniculata, Setaria poiretiana, Eichornia crassipes.

As espécies dicotiledôneas $(2,5,6,7$, 13,14,16,22) são: Ageratum conyzoides, Eclipta alba, Erechitites hieracifolia, E. valerianaefolia, Galinsoga parviflora, Gnaphalium spathulatum, Sonchus oleraceus, Cuphea carthagenensis, Ludwigia laruotteana, L. longifolia, L. sericea, Polygonum acre, P. hidropiperoides, Portulacca oleracea.

Das espécies monocotiledôneas com ciclo anual e perene observamos que as épocas de florescimento e frutificação são predominantes nos meses de setembro a março.

As dicotiledôneas anuais apresentamse com florescimento e frutificação durante todo o ano, ficando porém, caracterizado que o período de abundante florescimento e frutificação recai marcadamente nos meses de inverno e verão, enquanto que, as de ciclo perene não desenvolvem florescimento nos meses mais frios do ano.

\section{LITERATLRA CITADA}

1. Aranha. C.: Bacchi. O. \& Leitảo $F^{\prime \prime}$.. H.F. . Plantas Invasoras de Culturas no Estado de Sảo Paulo vol. III rem publicaçào.

2. Barroso. G.M. - Contribuiçáo ao estudo do gènero Cuphea Adans Rodriguesia 28-29: 193-212. 1954.

3. Barroso, G.M. - Flora da Cidade do Rio de Janeiro. Familia Compositae Rodriguesia 33-34: 69-115, 1953.

4. Barroso. L.J. - Chaves para identificaçào de gèneros indigenas e exóticos das Monocotiledóneas do Brasil Rodriguesia 20:55-77, 1946.

5. Bentham, G. \& Hooker. J.D. - Genera Plantarum vol. 1 p. 1040.1867.

6. Bentham. G. \& Hooker, J.D. - Genera Plantarum vol. 2 p. 1279.1876

7. Bentham. G. \& Hooker, J.D. - Genera Plantarum vol. 3 p. 1285.1883

8. Cabrera.A.L. Compuestas bonariensis Revista del Museo de La Plata Nueva serie Tomo 4. 450p.. 1941.

9. Camargo, P.N. et alii - Texto básico de contróle químico de plantas daninhas Apostila da Escola Superior de Agricultura "Luiz de Queiróz ${ }^{*}$ da Universidade de São Paulo $2^{\mathrm{a}} \mathrm{ed}$.. 257p., 1970.

10. Crovetto, R.M. \& Piccinni. B.G. - Bibliografia Argentina sobre malezas Publicacion Técnica $\mathrm{n}^{\circ}$ 17. Minist. da Agricultura 91p., 1948.

11. Crovetto, R.M. - Las malezas de los cespedes em la capital federal $\boldsymbol{y}$ alrededores Publica. cion Técnica $n^{\circ} 41.46 \mathrm{p} . .1950$.

12. Crovetto, R.M. - Las malezas de los montes frutales en el nordeste de Entre Rios Publica. cion Tecnica $\mathrm{n}^{*}$ 54, 44p., 1950.

13. Ducke. A.J. - Amaranthaceae Fiora of Pantma Annals of the Missouri Botanical Garden 48:6-50, 1961

14. Freire, C.V. - Chaves Analiticas Disi 1.11 Kosmos, 366p. 1943.

15. Font Quer. P. - Diccionario de Botanica E.t: Labor S A 1244p.. 1953

16. Gemtchujnicov. I. - Chave artifical para identificaçao de plantas daninhas do Estadode Sato Paulo Apostila mimeografada FCME E. 266p., 1968.

i7. Hitcheock. A.S. - The North American Spe cies of Echinochloa Contributions fron. the United States National Herbariun: 22131.133154. 1920

18. Hitcheock. A.S. - Grasses of British Guiana $22161,439 \cdot 514,1922$

19. Hitcheock. A.S - A Text-book of Grasses TheMacmillan Company $276 \mathrm{p} ., 1922$

20. Isely, D. - Weed identification and control Iowa State University Press Ames. Iok a U.S.A. 400p. 1962.

21. Lagos, J.A. \& Calles, F. - Malas hierbas en cafetales de El Savador Facultad de Cienc. Agron. Bol. n' 2 36p', 1968.

22. Leitáo $\mathbf{F}^{\prime}$. H.F. - Especies de Amarant: què ocorrem como invasoras no muncep.o at Campinas Bragantia 27:476-492, 1968 .

23. Leituo F H.F. \& Aranha. C - Noces stis: sistematica de plantas invasoids ES A litir de Queiróz" 44p.. 1970

24. Leituo F ${ }^{*}$.. H.F.. Aranha. C \& Baceh. Q Plantas Invasoras de Culturas no Estado de Sào Paulo HUCITEC Sao Patio vol I :2! ? 1972

25. Leitào $F^{v}$. H F. Aranha. C \& Batch o Plantas Invasoras de Culturas no Estado de Sảo Paulo HUCITEC Sào Pauio Vol II 597, 1975

26. Munz. P.A. - Studies in Onagracese A wes. sion of the New Worid species of Jussiaed Darwiniana 4:179-284. 194: 